\title{
Auroral evidence of Io's control over the magnetosphere of Jupiter
}

\author{
B. Bonfond, ${ }^{1}$ D. Grodent, ${ }^{1}$ J.-C. Gérard, ${ }^{1}$ T. Stallard, ${ }^{2}$ J. T. Clarke, ${ }^{3}$ M. Yoneda, ${ }^{4}$ \\ A. Radioti, ${ }^{1}$ and J. Gustin ${ }^{1}$ \\ Received 3 November 2011; revised 28 November 2011; accepted 2 December 2011; published 11 January 2012.
}

[1] Contrary to the case of the Earth, the main auroral oval on Jupiter is related to the breakdown of plasma corotation in the middle magnetosphere. Even if the root causes for the main auroral emissions are Io's volcanism and Jupiter's fast rotation, changes in the aurora could be attributed either to these internal factors or to fluctuations of the solar wind. Here we show multiple lines of evidence from the aurora for a major internally-controlled magnetospheric reconfiguration that took place in Spring 2007. Hubble Space Telescope far-UV images show that the main oval continuously expanded over a few months, engulfing the Ganymede footprint on its way. Simultaneously, there was an increased occurrence rate of large equatorward isolated auroral features attributed to injection of depleted flux tubes. Furthermore, the unique disappearance of the Io footprint on 6 June appears to be related to the exceptional equatorward migration of such a feature. The contemporary observation of the spectacular Tvashtar volcanic plume by the New-Horizons probe as well as direct measurement of increased Io plasma torus emissions suggest that these dramatic changes were triggered by Io's volcanic activity. Citation: Bonfond, B., D. Grodent, J.-C. Gérard, T. Stallard, J. T. Clarke, M. Yoneda, A. Radioti, and J. Gustin (2012), Auroral evidence of Io's control over the magnetosphere of Jupiter, Geophys. Res. Lett., 39, L01105, doi:10.1029/2011GL050253.

\section{Introduction}

[2] The Jovian magnetosphere is supplied by a permanent internal source of plasma: Io's volcanism. Created by the sublimation of $\mathrm{SO}_{2}$ frost of volcanic origin, Io's tenuous atmosphere releases around 1 ton/second of $\mathrm{SO}_{2}$ in the form of a neutral cloud. After being ionized through collisions or photo-ionization, half of this material escapes the magnetosphere as energetic neutral atoms via charge exchange, while the remaining half further populates the plasma torus along Io's orbit [Thomas et al., 2004]. The plasma cannot indefinitely accumulate in this torus and it migrates radially through flux tube interchange driven by centrifugal instability. As the plasma moves outward, the magnetic field lines evolve from a

\footnotetext{
${ }^{1}$ Laboratoire de Physique Atmosphérique et Planétaire, Université de Liège, Liège, Belgium.

${ }^{2}$ Department of Physics and Astronomy, University of Leicester, Leicester, UK.

${ }^{3}$ Center for Space Physics, Boston University, Boston, Massachusetts, USA.

${ }^{4}$ Planetary Plasma and Atmospheric Research Center, Graduate School of Science, Tohoku University, Sendai, Japan.

Copyright 2012 by the American Geophysical Union. 0094-8276/12/2011GL050253
}

dipolar to a more stretched sheet-like configuration. Initially, this escaping plasma keeps corotating rigidly with the planet; the additional momentum is provided by Jupiter's ionosphere through electric currents along the magnetic field lines. But this process cannot be pursued indefinitely, and models show that these currents peak just before the distance where corotation breaks down, generating the intense main auroral emissions on Jupiter [Hill, 2001]. Conservation of the magnetic flux imposes that, while heavy flux tubes progressively move outwards, depleted flux tubes should move inwards to replace them. Indeed, evidences of small scale flux tube interchange have been found in the Io torus [Bolton et al., 1997; Kivelson et al., 1997; Thorne et al., 1997]. On the other hand, much larger injections of hot but sparse plasma have been observed all around Jupiter between 9 and $27 \mathrm{Rj}$ and their auroral signatures have been identified as isolated auroral features, also referred to as blobs [Mauk et al., 1999, 2002]. The link between flux tube interchange and injections is not clear yet, but Russell et al. [2005] noted that the depleted flux tubes tend to group into bunches, a possible hint of filamentation of larger structures.

[3] A large Hubble Space Telescope (HST) campaign dedicated to Jupiter's far-UV aurora was carried out in Spring 2007 with the Solar Blind Channel of the Advanced Camera for Surveys (ACS). For the first time, this quasidaily coverage of the aurora over 5 months provided a large body of evidence demonstrating that major reconfigurations of the Jovian magnetosphere are internally driven.

\section{Observations}

\subsection{Location of the Ganymede Auroral Footprint}

[4] Figure 1 (left) shows a polar projection of the northern aurora on 27 February 2007. The Ganymede footprint is located on the left side of the image, corresponding to the dawn side of the planet. The System III (S3) longitude of Ganymede was $246.6^{\circ}$ and its phase angle was $88.7^{\circ}\left(0^{\circ}\right.$ corresponds to midnight, $90^{\circ}$ to dawn). The Ganymede footprint was located at $201.2^{\circ}$ in System III longitude and $60.5^{\circ}$ in planetocentric latitude. As usual, the footprint is located equatorward of the main emission. Figure 1 (right) shows a projection of the same hemisphere in a very similar configuration - Ganymede was at $247.0^{\circ}$ System III longitude and $92.7^{\circ}$ phase angle - but on 31 May 2007. The Ganymede footprint shifted $\sim 500 \mathrm{~km}$ equatorward $\left(202.0^{\circ} \mathrm{S} 3\right.$ lon and $60.3^{\circ}$ lat). However the most striking difference involves the main emission dawn branch, which is now located $\sim 3000 \mathrm{~km}$ equatorward (see Animation S1 in the auxiliary material). ${ }^{1}$

\footnotetext{
${ }^{1}$ Auxiliary materials are available in the HTML. doi:10.1029/ 2011 GL050253.
} 

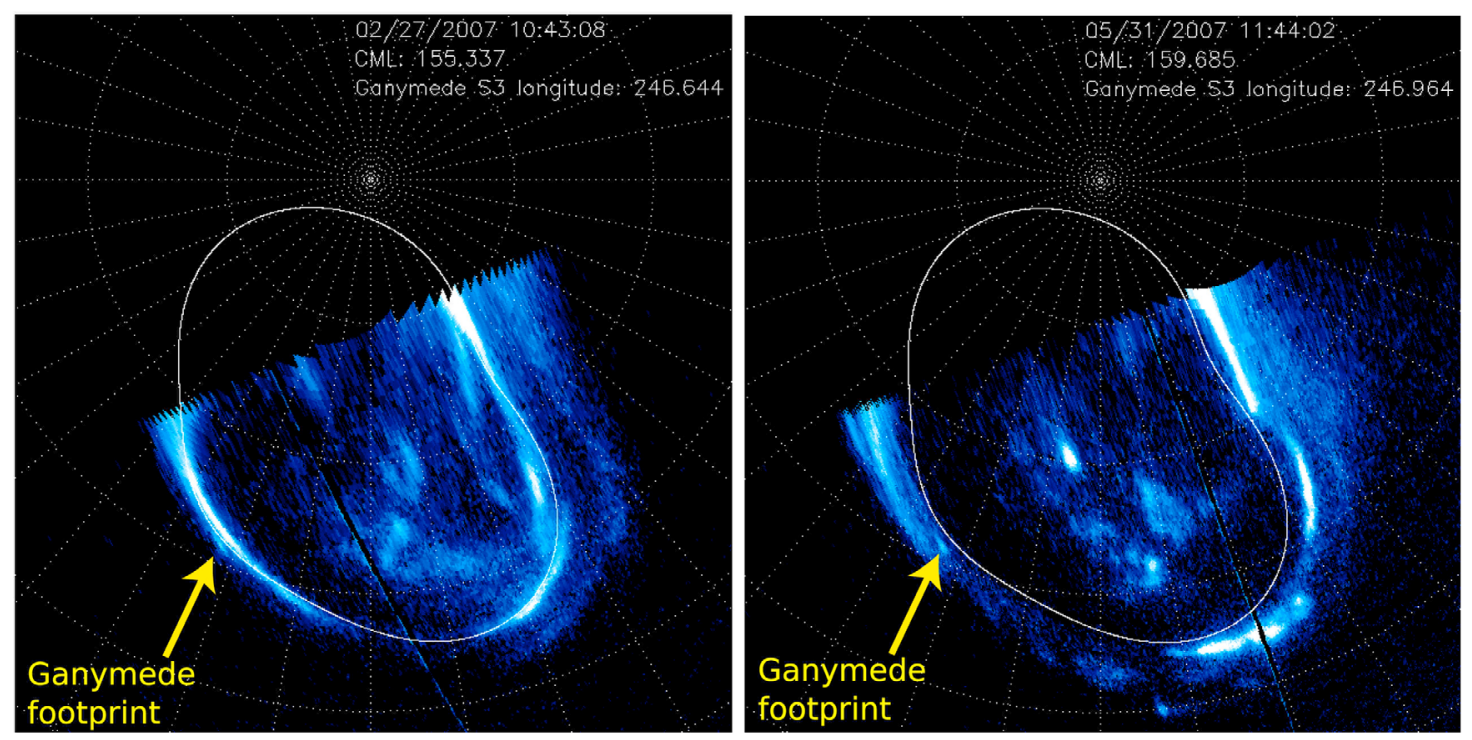

Figure 1. Polar projection of the northern hemisphere aurora (left) on February 27th and (right) on May 21st 2007. The observing geometry was very similar, with CMLs of $155.3^{\circ}$ and $159.7^{\circ}$ respectively and a Ganymede S3 longitude of $246.6^{\circ}$ and $247.0^{\circ}$ respectively. Nevertheless, the Ganymede footprint is outside the main emission in the first image and inside it in the second case, suggesting that the corotation breakdown boundary has moved inside the Ganymede orbit (15 Rj). Additionally, the Ganymede footprint location moved $0.5^{\circ}$ equatorward implying an increased stretching of the magnetic field lines. The white line is the reference oval from February 2007.

\subsection{Motion of the Main Auroral Emissions}

[5] The expansion of the main oval on 31 May is not an isolated event, but is part of a continuous increase of the main oval size observed from February to June 2007. Polar projections of the aurora have been co-added in order to create monthly maps for both hemispheres. We do not consider the April map of the northern hemisphere in this study because of large gaps resulting from a lack of observations during this period. The size and shape of the February main oval in the northern hemisphere are very similar to those inferred from previous ACS observations acquired in 2005 and 2006. However, the size of the main oval in the southern hemisphere shows a continuous mean radial expansion ultimately reaching $\sim 1700 \mathrm{~km}$ (see Figure 2 and Animations S2 and S3), which would correspond to $\sim 2^{\circ}$ of latitude if the magnetic field were axisymmetric. A similar calculation in the northern hemisphere gives half the value, owing to the combination of the incomplete longitude coverage in the North and to the north-south magnetic field asymmetry. The magnetic flux contained inside the southern main oval increased by $\sim 250 \mathrm{GWb}$ from February to June 2007, according to the VIPAL magnetic field model [Hess et al., 2011].

[6] Figure 2 also shows that transient variations of the main oval size are superimposed on the long term trend. These shorter timescale variations are probably related to compressions of the magnetosphere associated with the solar wind pressure [Nichols et al., 2009]. Indeed, compressions and expansions of the magnetopause due to variations of the solar wind parameters are expected to influence the main oval size and brightness [Cowley et al., 2007]. However, such changes would be correlated with the solar wind conditions and should thus take place on timescales ranging from hours to days. Solar wind parameters obtained either through

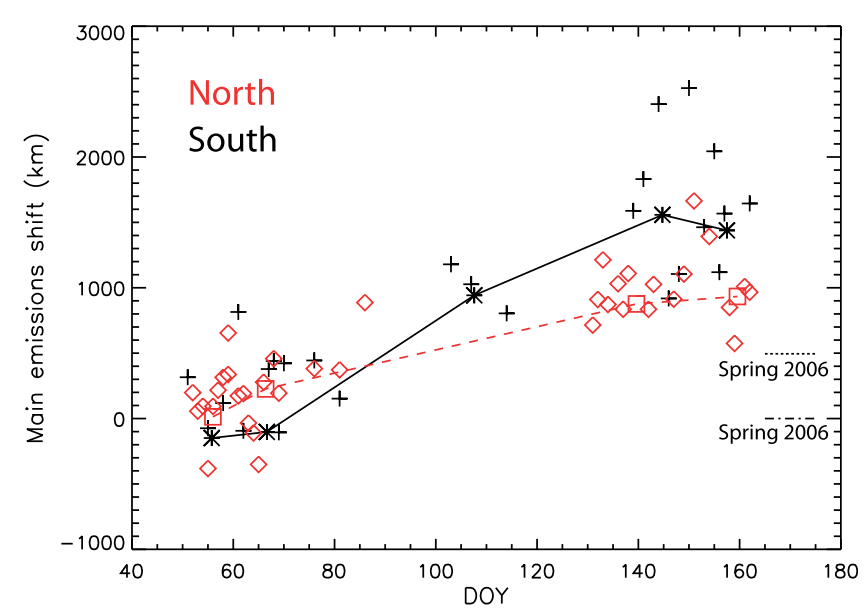

Figure 2. Evolution of the mean distance between the main emissions and a reference oval made of a 4 th order Fourier fit of the February 2007 data. This reference period has been preferred to former ones [e.g., Grodent et al., 2003] because it allowed a fairly complete S3 coverage of the two poles within only two weeks. Black crosses and red diamonds represent daily observations for the South and for the North respectively. The black stars linked by the black solid line and the red squares linked by the red dashed line represent the monthly averages in the southern and northern hemispheres, respectively. They display a continuous and significant increase over the months. The North/South discrepancy is owing to the incomplete longitude sampling in the $330^{\circ}-20^{\circ} \mathrm{S} 3$ sector in the North and to the stronger magnetic field magnitude in the northern hemisphere around $160^{\circ} \mathrm{S} 3$. The horizontal dash-dotted and the dotted lines represent the average shifted distance for the 2005 and 2006 northern hemisphere campaigns, respectively. 

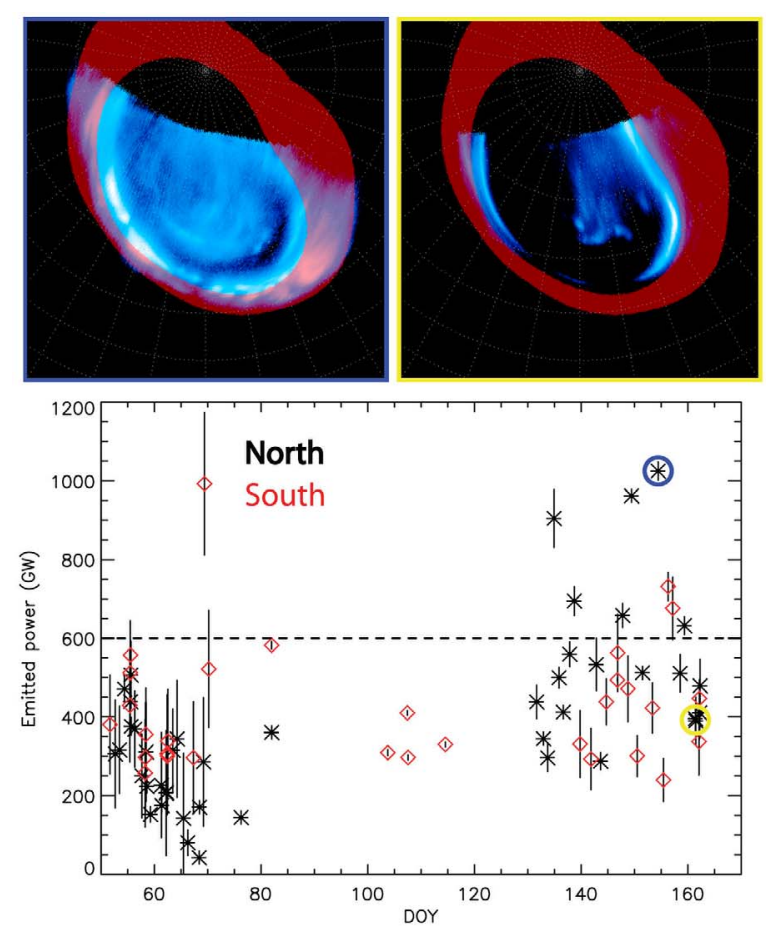
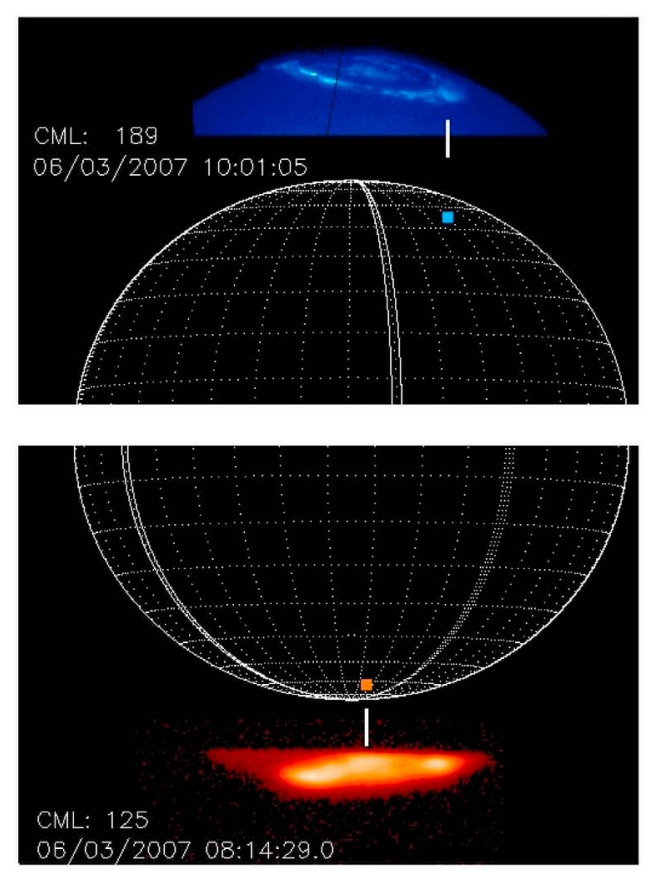

Figure 3. (bottom left) Evolution of the emitted power of the outer auroral emission. Black stars and red diamonds represent the mean power over 45 minute long sequences for the North and for the South respectively. The power is integrated over a ribbon starting $\sim 1200 \mathrm{~km}$ equatorward of the monthly averaged main oval and ending at the Io footpath. The plotted values are corrected to account for the ratio between the total ribbon surface and the visible one. The error bars represent the dispersion of the measured power over the sequence, essentially owing to the appearance or disappearance of features as Jupiter rotates. The number of cases with a total outer emissions power over $600 \mathrm{GW}$ significantly increased in May-June. (top left) Polar projections corresponding to the encircled points in Figure 2 (bottom left) with the outer emissions ribbon shown in red. The left one (in blue) shows a case with bright patches of outer emissions while the right one (in yellow) shows a quiet case. (right) Two images acquired less than 2 hours apart in the IR domain with the IRTF in the southern hemisphere on the bottom side and in the UV domain with the Hubble Space Telescope in the northern hemisphere on the top side. On each side, the white line underlines the large auroral blob which is tracked from one hemisphere to the other.

direct measurement by the New-Horizons probe or through solar wind propagation models from the Earth to Jupiter do not show any clear long-term trend from February to June [Clarke et al., 2009].

\subsection{Variability of Outer Emissions}

[7] In addition to the main oval expansion, the May-June campaign shows an increased occurrence of particularly large patches of UV and infrared emission between the main emission and the Io footpath. These features are usually associated with injections of hot plasma coming from the outer magnetosphere [Mauk et al., 2002]. Figure 3 shows the integrated power emitted in a ribbon located between the outer edge of the main emission and the Io footpath. Since the mean location of the main emissions evolved during the campaign, we used a monthly reference oval for the poleward boundary. The displayed emitted power has been corrected for the viewing geometry by a factor corresponding to the ratio between the visible surface and the total surface of the ribbon. Only cases with a correction factor less than 2 have been considered in order to avoid unreasonably large extrapolations of the emissions in the region of interest. Unusually large emissions $(>600 \mathrm{GW})$ are seen 8 times in May and June, while only one occurrence has been seen in
March. Contemporary infrared observations acquired with the IRTF telescope in Hawaii over the same time period confirm the presence of these large blobs during the same time interval (Figure 3 and Animation S4).

\subsection{Unusual Io UV Footprint Behavior}

[8] The equatorward blobs are usually confined between the main emissions and the Io footpath. However on 7 June 2007 , a large $(\sim 15000$ by $\sim 6000 \mathrm{~km})$ patch of emission is seen down to the expected location of the Io footprint (Figure 4 as well as Animations S5 and S6). This patch appears to be the remnant of a large injection blob seen in the same sector in the southern hemisphere 34 hours before. At the beginning of the sequence, the Io footprint should emerge from the patch, but cannot be distinguished from the background emissions. The maximum apparent brightness from the location where we would expect the Io spot is estimated to be below $200 \mathrm{kR}$ in $\mathrm{H}_{2}$ Lyman and Werner bands, corresponding to an emitted power of less than $1 \mathrm{GW}$ for the main spot. The Io footprint can experience brightness variations of a factor 2 within a few minutes [Bonfond et al., 2007]. Nevertheless this case starkly differs from all other 38 similar observations with Io S3 longitude between $202^{\circ}$ and $212^{\circ}$ and carried out between June 1999 and May 2007, 

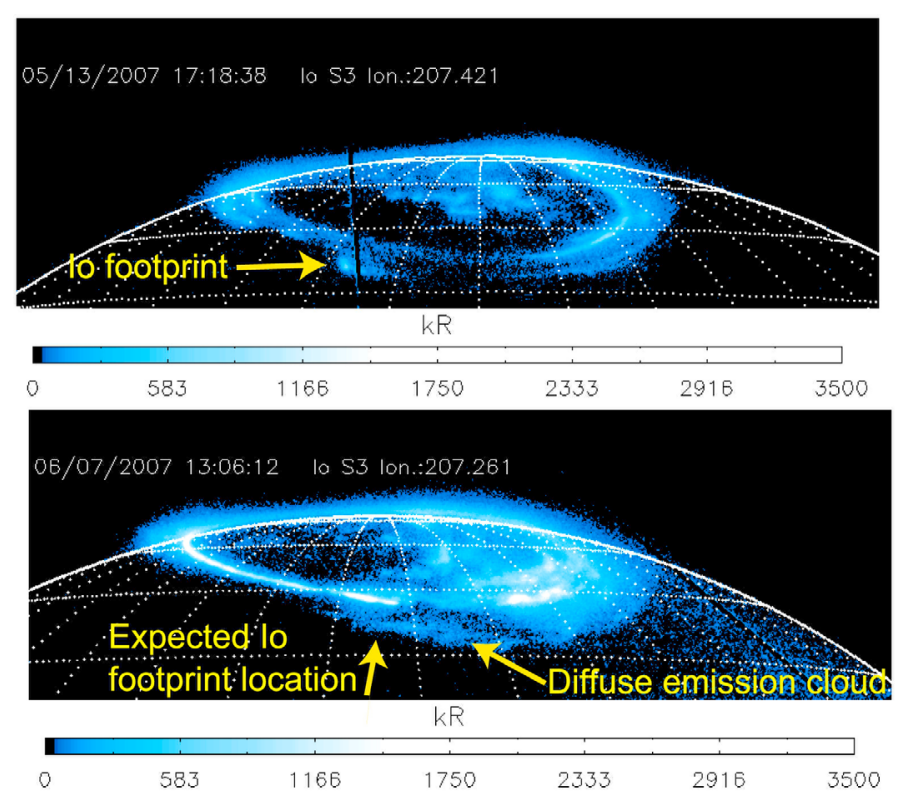

Figure 4. (top left) HST ACS images of the northern hemisphere in two very similar geometries. (bottom left) While Io's S3 longitude is nearly the same, the Io footprint is not visible, while it would be expected to lie at the border of the unusually equatorward patch of diffuse emission. This emission patch appears to be the remnant of a large injection blob seen 34 hours before in the southern hemisphere, (right) as shown on the polar projection. This disappearance of the Io footprint is unique within more than 10 years of high-resolution/high sensibility HST images and may be due to a disrupted interaction between Io and the depleted flux tubes connected to the patch.

which show a northern Io footprint main spot between $\sim 3$ and $\sim 6.5 \mathrm{GW}$.

\section{Discussion and Conclusions}

[9] Previously observed variations of the main oval position were attributed either to reconfiguration of the current sheet or to solar-wind induced magnetospheric compressions [Grodent et al., 2003, 2008; Nichols et al., 2009]. Grodent et al. [2008] compared two images of the northern hemisphere aurora acquired in quasi-identical configurations but 5 years apart. They noted that the main emission moved $3^{\circ}$ poleward and the Ganymede footprint shifted by $2^{\circ}$ in the same direction. Using the plasma sheet magnetic field model from Connerney [1981], they concluded that variations of the azimuthal current by a factor of 3 could sufficiently stretch the field lines to account for the observations. Unfortunately, these observations took place on two isolated days, which prevents disentangling day-by-day variations from long-term trends.

[10] Motion of the main emission location could be attributed either to changes in the magnetic field stretching or to a displacement of the corotation breakdown boundary. However, if the dawn branch of the main emission in Figure 1 actually corresponds to the corotation breakdown boundary, the reversal of location implies that the latter moved inside the orbit of Ganymede. According to theoretical models [Hill, 2001; Nichols, 2011; Ray et al., 2012], the position of the breakdown boundary depends either on the mass outflow rate, on the ionospheric conductivity, or on the expansion/compression of the magnetopause. Only the first explanation has an evolution timescale of few weeks [Delamere et al., 2005], compatible with the observations. Our suggested scenario is that Io's volcanism became particularly active in February 2007, as evidenced by the spectacular Tvashtar plume seen by New-Horizons [Spencer et al., 2007]. Strong volcanic activity is then expected to have continued at least intermittently, as demonstrated by the tripling of the Io torus sodium nebula brightness observed by Yoneda et al. [2009] in late May. In this scenario, the progressive ionization of volcanic material released by Io increased the density of the plasma sheet, increasing the azimuthal current and thus moving the Ganymede footprint equatorward. It also strengthened the mass outflow rate, forcing the corotation breakdown to occur closer to Jupiter, which further expanded the main auroral oval. Hill [2001] used a dipole to model the magnetic field and estimated that a quadrupled mass outflow would explain the $\sim 2^{\circ}$ expansion. However, recent models considering a more realistic magnetic field stretching due to the plasma sheet require a mass outflow rate $>10$ times stronger to achieve the same result [Nichols, 2011; Ray et al., 2012]. It is nevertheless unlikely that an iogenic outburst could modify the mass outflow rate only. Other quantities such as the electron temperature or the Pedersen conductivity are also expected to vary and further theoretical work is required to pinpoint the suitable set of parameters to reproduce the oval expansion described here.

[11] The large amount of outward moving heavy flux tubes has to be replaced by flux tubes sparsely filled with hot plasma. The enhanced loading of the middle magnetosphere could then explain the highest occurrence rate of large features associated with injection signatures in May-June compared to February-March. We suggest that on 7 June, a large cloud of depleted flux tubes migrated exceptionally close to Jupiter and disrupted the Io-Jupiter interaction, begetting an abnormally faint Io footprint. 
[12] Acknowledgments. The authors would like to thank Licia Ray and Jonathan Nichols for helpful discussions. B.B. was supported by the PRODEX program managed by ESA in collaboration with the Belgian Federal Science Policy Office. J.C.G., D.G. and A.R. are funded by the Belgian Fund for Scientific Research (FNRS). This research is based on observations made with the Hubble Space Telescope obtained at the Space Telescope Science Institute, which is operated by AURA Inc. Work at BU was supported by grant HST-GO-11649.01-A from STScI to Boston University.

[13] The Editor thanks two anonymous reviewers for their assistance in evaluating this paper.

\section{References}

Bolton, S. J., R. M. Thorne, D. A. Gurnett, W. S. Kurth, and D. J. Williams (1997), Enhanced whistler-mode emissions: Signatures of interchange motion in the Io torus, Geophys. Res. Lett., 24, 2123-2126, doi:10.1029/ 97GL02020.

Bonfond, B., J.-C. Gérard, D. Grodent, and J. Saur (2007), Ultraviolet Io footprint short timescale dynamics, Geophys. Res. Lett., 34, L06201, doi:10.1029/2006GL028765.

Clarke, J. T., et al. (2009), Response of Jupiter's and Saturn's auroral activity to the solar wind, J. Geophys. Res., 114, A05210, doi:10.1029/ 2008JA013694.

Connerney, J. E. P. (1981), The magnetic field of Jupiter: A generalized inverse approach, J. Geophys. Res., 86, 7679-7693, doi:10.1029/ JA086iA09p07679.

Cowley, S. W. H., J. D. Nichols, and D. J. Andrews (2007), Modulation of Jupiter's plasma flow, polar currents, and auroral precipitation by solar wind-induced compressions and expansions of the magnetosphere: A simple theoretical model, Ann. Geophys., 25, 1433-1463, doi:10.5194/ angeo-25-1433-2007.

Delamere, P. A., F. Bagenal, and A. Steffl (2005), Radial variations in the Io plasma torus during the Cassini era, J. Geophys. Res., 110, A12223, doi:10.1029/2005JA011251.

Grodent, D., J. T. Clarke, J. Kim, J. H. Waite Jr., and S. W. H. Cowley (2003), Jupiter's main auroral oval observed with HST-STIS, J. Geophys. Res., 108(A11), 1389, doi:10.1029/2003JA009921.

Grodent, D., J.-C. Gérard, A. Radioti, B. Bonfond, and A. Saglam (2008), Jupiter's changing auroral location, J. Geophys. Res., 113, A01206, doi:10.1029/2007JA012601.

Hess, S. L. G., B. Bonfond, P. Zarka, and D. Grodent (2011), Model of the Jovian magnetic field topology constrained by the Io auroral emissions, J. Geophys. Res., 116, A05217, doi:10.1029/2010JA016262.

Hill, T. W. (2001), The Jovian auroral oval, J. Geophys. Res., 106, 8101-8107, doi:10.1029/2000JA000302.

Kivelson, M. G., K. K. Khurana, C. T. Russell, and R. J. Walker (1997), Intermittent short-duration magnetic field anomalies in the Io torus: Evidence for plasma interchange?, Geophys. Res. Lett., 24, 2127-2130.
Mauk, B. H., D. J. Williams, R. W. McEntire, K. K. Khurana, and J. G. Roederer (1999), Storm-like dynamics of Jupiter's inner and middle magnetosphere, J. Geophys. Res., 104(A10), 22,759-22,778, doi:10.1029/ 1999JA900097.

Mauk, B. H., J. T. Clarke, D. Grodent, J. H. Waite, C. P. Paranicas, and D. J. Williams (2002), Transient aurora on Jupiter from injections of magnetospheric electrons, Nature, 415, 1003-1005.

Nichols, J. D. (2011), Magnetosphere-ionosphere coupling in Jupiter's middle magnetosphere: Computations including a self-consistent current sheet magnetic field model, J. Geophys. Res., 116, A10232, doi:10.1029/ 2011JA016922.

Nichols, J. D., J. T. Clarke, J. C. Gérard, D. Grodent, and K. C. Hansen (2009), Variation of different components of Jupiter's auroral emission, J. Geophys. Res., 114, A06210, doi:10.1029/2009JA014051.

Ray, L. C., R. E. Ergun, P. A. Delamere, and F. Bagenal (2012), Magnetosphere-Ionosphere coupling at Jupiter: A parameter space study, J. Geophys. Res., doi:10.1029/2011JA016899, in press.

Russell, C. T., M. G. Kivelson, and K. K. Khurana (2005), Statistics of depleted flux tubes in the Jovian magnetosphere, Planet. Space Sci., 53, 937-943, doi:10.1016/j.pss.2005.04.007.

Spencer, J. R., et al. (2007), Io volcanism seen by New Horizons: A major eruption of the Tvashtar Volcano, Science, 318, 240-243, doi:10.1126/ science. 1147621 .

Thomas, N., F. Bagenal, T. W. Hill, and J. K. Wilson (2004), The Io neutral clouds and plasma torus, in Jupiter: The Planet, Satellites and Magnetosphere, edited by F. Bagenal, T. E. Dowling, and W. B. McKinnon, pp. 561-591, Cambridge Univ. Press, Cambridge, U. K

Thorne, R. M., T. P. Armstrong, S. Stone, D. J. Williams, R. W. McEntire, S. J. Bolton, D. A. Gurnett, and M. G. Kivelson (1997), Galileo evidence for rapid interchange transport in the Io torus, Geophys. Res. Lett., 24, 2131-2134, doi:10.1029/97GL01788.

Yoneda, M., M. Kagitani, and S. Okano (2009), Short-term variability of Jupiter's extended sodium nebula, Icarus, 204, 589-596, doi:10.1016/j. icarus.2009.07.023.

B. Bonfond, J.-C. Gérard, D. Grodent, J. Gustin, and A. Radioti, Laboratoire de Physique Atmosphérique et Planétaire, Université de Liège, 17, Allée du 6 Août, B-4000 Liège, Belgium. (b.bonfond@ulg.ac.be) J. T. Clarke, Center for Space Physics, Boston University, 725 Commonwealth Ave., Boston, MA 02215, USA.

T. Stallard, Department of Physics and Astronomy, University of Leicester, University Road, Leicester LE1 7RH, UK.

M. Yoneda, Planetary Plasma and Atmospheric Research Center, Graduate School of Science, Tohoku University, 6-3 Aramaki-aza-aoba, Aoba-ku, Sendai, Miyagi 980-8578, Japan. 